\title{
Amélioration de la stabilité de fonctionnement des turbines Francis par contrôle actif - Etude de faisabilité
}

\author{
Improving the Operation Stability of Francis Turbines \\ by Active Control - A Feasibility Study \\ par G. Blommaert, J.-E. Prénat, F. Avellan \\ EPFL-IMHEF-LMH, Lausanne \\ A. Boyer, J.-F. Lauro \\ EDF-DER Chatou
}

Francis turbines, operating at off-design conditions, generate hydraulic disturbances in the hydraulic circuit. In certain cases, the amplitude of the dynamic response of the system to these excitations can inhibit normal usage of the plant.

One approach to reduce the amplitude fluctuations is to deliberately excite the system with the inverse signal of the naturally generated disturbances. The idea is to minimize the overall amplitude of the fluctuations by actively controlling the system. Experimental results were obtained with a rotating valve exciter mounted on a laboratory test rig equipped with a Francis furbine. The actuator modulates the flow injected into the circuit. The external excitation system has been tested at different places of water intake and injection. The results confirm that a small excitation flow can significantly reduce the amplitude of pressure fluctuations transmitted to the feed pipe.

\section{INTRODUCTION}

Le fonctionnement des machines hydrauliques génère souvent des perturbations hydrauliques dans les circuits environnants. Dans certains cas - par exemple les turbines Francis hors de leur régime optimal - la réponse dynamique du système à ces perturbations peut atteindre une amplitude telle qu'elle empêche l'exploitation normale de l'installation.

La recherche en ce domaine est surtout dirigée vers la prédiction et la prévention $[3,1]$ des instabilités hydrauliques, l'idée générale étant de produire des tracés de roues et d'aspirateurs qui minimisent les excitations. Les techniques de correction [1] impliquant de petites modifications de la machine forment un autre sujet de recherche.

Ces méthodes sont mises en œuvre quand le fonctionnement d'une turbine se révèle instable à sa mise en service.

Le contrôle actif consiste en un réglage d'un paramètre physique d'un système dynamique au moyen d'un apport d'énergie externe. Une solution est d'exciter délibérément le système avec le signal inverse des perturbations émises naturellement. Le but est de minimiser le niveau global des fluctuations afin de rendre possible le fonctionnement des machines hydrauliques dans les régimes où des instabilités hydrauliques obligent pour le moment à une utilisation conditionnelle [2].

\section{II $\square$ OBJECTIFS}

Les objectifs de ces essais de faisabilité sur modèle sont : — de montrer l'efficacité d'un système de contrôle actif pour la réduction des instabilités des turbines Francis, aussi bien au niveau hydraulique (pression et débit) qu'au niveau mécanique (couple et vitesse de rotation),

- de vérifier le fonctionnement correct d'un actionneur de type vanne rotative,

- de connaître l'influence de la position de l'actuateur,

- d'évaluer la puissance consommée par un tel système afin d'obtenir des éléments pour dimensionner un système adapté à une turbine prototype.

\section{III — DISPOSITIF EXPÉRIMENTAL}

\section{- 3.1 Concept}

Bien que la physique des phénomènes aéro- et hydroacoustiques repose sur les mêmes bases, les éléments critiques sont très différents.

Pour un bruit aérien, l'amplitude des fluctuations de pression est très petite, tant en valeur absolue qu'en valeur relative à la pression atmosphérique. Un simple haut-parleur suffit comme source secondaire pour générer un "contrebruit" afin d'annuler le bruit d'une source primaire. Dans le 


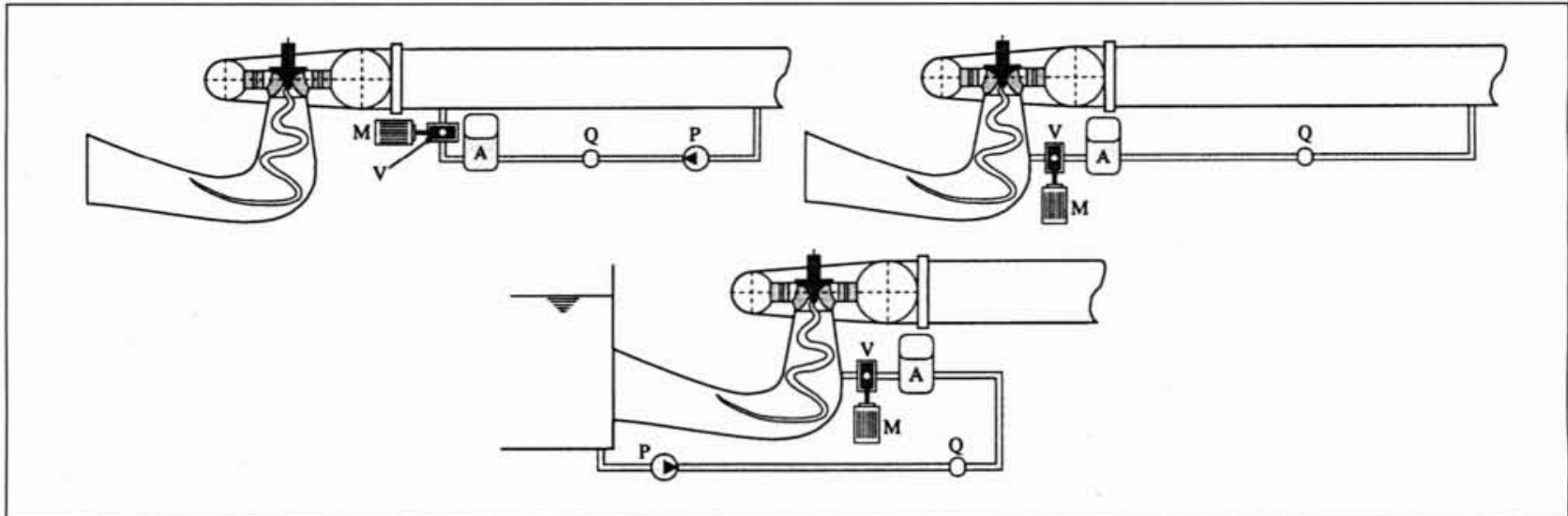

1. Les trois configurations des essais effectués.

cas des machines hydrauliques par contre, l'amplitude des fluctuations de pression absolue et relative à la pression moyenne locale peut être importante. Cela implique de disposer d'un actionneur approprié afin de générer une puissance acoustique suffisante pour compenser l'excitation naturelle de la turbine. L'équivalent d'un haut-parleur serait un piston monté en paroi de la conduite.

Un tel dispositif a quelques inconvénients :

- pour pouvoir générer une puissance acoustique suffissante, les dimensions du dispositif deviennent très importantes, limitant la plage de fonctionnement à des fréquences très basses ;

— il se pose également un problème d'entraînement : l'énergie réactive nécessaire pour accélérer et décélérer la masse du piston est grande et implique un moteur puissant.

Les essais de faisabilité [2] ont montré qu'une excitatrice de type vanne rotative ou vanne oscillante contourne ces inconvénients et offre une solution envisageable. Les dimensions de l'excitatrice restent petites parce que la puissance est fournie par une pompe d'injection et non par le mouvement d'une pièce mécanique.

\subsection{Description de l'excitatrice}

L'actionneur (figure 1) est une vanne rotative (V) : une vanne sphérique ayant un corps cylindrique. Elle est entraînée par un moteur à vitesse variable $(\mathrm{M})$ et permet de moduler le débit qu'injecte une pompe en dérivation $(\mathrm{P})$. Le débit moyen d'excitation est mesuré par un compteur d'eau (Q). L'excitatrice est raccordée par des tuyaux souples. Un accumulateur à air $(\mathrm{A})$ découple dynamiquement la pompe d'injection - et donc également le circuit d'essai - de l'actionneur.

L'alésage de la vanne est $\phi 80 \mathrm{~mm}$. Un signal en tension commande la vitesse de rotation du moteur. L'amplitude de l'excitation externe est ajustée manuellement à l'aide d'une vanne à l'entrée de l'accumulateur. Cet ensemble constitue l'excitatrice.

\section{- 3.3 Pilotage de l'actionneur}

Le moteur de la vanne rotative est piloté en vitesse par l'intermédiaire d'une carte d'acquisition AT-MIO-16XE-10 de National Instruments, aisément programmable avec le logiciel LabVIEW.

Les fluctuations de pression dans une turbine Francis, dues à un mouvement de précession de l'écoulement dans le cône de l'aspirateur pour les régimes de charge partielle, sont caractérisées par la présence d'une fréquence prépondérante. Cette fréquence vaut environ 0,3 fois la fréquence de rotation de la turbine. L'approche finalement mise en œuvre joue sur la variation de la vitesse de rotation de l'excitatrice autour d'une vitesse fixe correspondant à cette fréquence prépondérante. Le but est de garder un rapport de phase entre l'excitation hydraulique due à la perturbation et l'excitation par la vanne rotative, qui minimise les fluctuations de pression globales.

\subsection{Montage d'essai}

Trois configurations différentes de l'excitatrice ont été testées (figure 1):

- avec actionneur à l'amont et prise d'eau à l'amont avec pompe d'injection,

- avec actionneur à l'aval et prise d'eau à l'amont sans pompe d'injection,

- avec actionneur à l'aval et prise d'eau à l'aval avec pompe d'injection.

Le circuit d'essai comporte un modèle réduit de turbine Francis qui présente de fortes fluctuations de pression à charge partielle. La turbine, dont la roue a un diamètre de sortie de $409 \mathrm{~mm}$, a une puissance nominale de $130 \mathrm{~kW}$. Le diamètre intérieur de la conduite d'alimentation est de $587 \mathrm{~mm}$.

Le point de fonctionnement (tableau 1) a été choisi à partir d'un essai de stabilité de fonctionnement : $76 \%$ du débit nominal et $100 \%$ de l'énergie hydraulique massique nominale.

\begin{tabular}{|l|c|c|c|c|}
\hline $\mathrm{Q}\left(\mathrm{m}^{3} / \mathrm{s}\right)$ & $\mathrm{E}(\mathrm{J} / \mathrm{kg})$ & $\mathrm{T}(\mathrm{Nm})$ & $\mathrm{NM}(\mathrm{tr} / \mathrm{min})$ & $\gamma$ \\
\hline 0,620 & 147 & 925 & 804 & $18,5^{\circ}$ \\
\hline
\end{tabular}

Tableau 1. - Les conditions de fonctionnement de la turbine.

Trois capteurs de pression piezoélectriques sont placés sur le cône de l'aspirateur et quatre dans la conduite d'alimentation, dont trois équidistants afin d'estimer la puissance acoustique globale. Les signaux sont enregistrés à l'aide d'un analyseur de spectres HP3566A et traités dans le domaine fréquentiel par le biais du logiciel Matlab. 


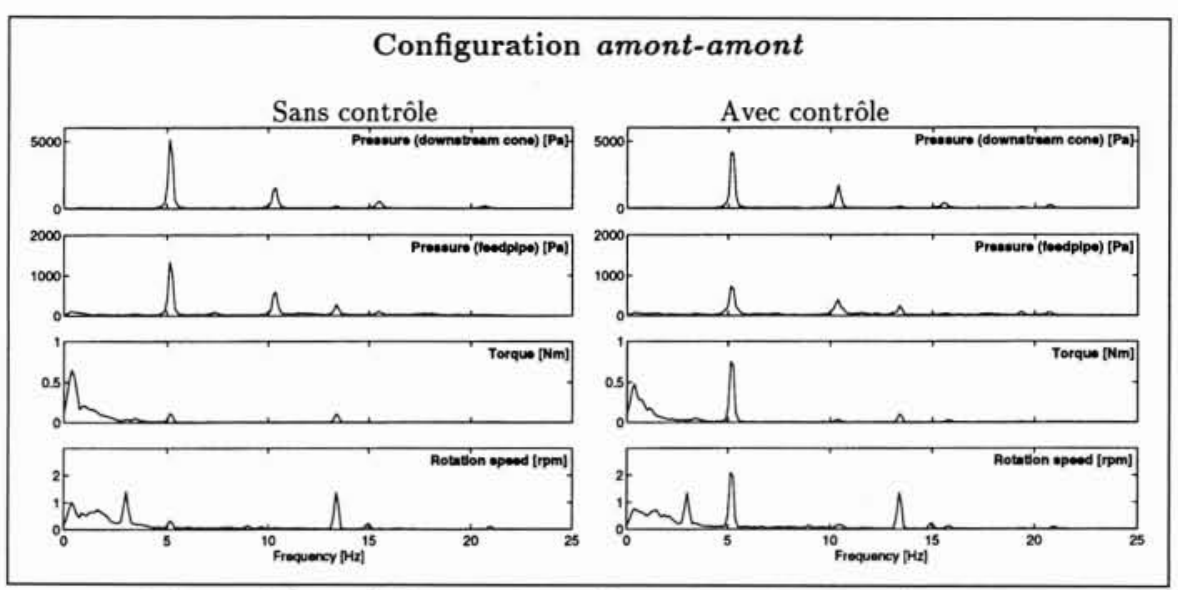

2. Trace fréquentielle pour la configuration prise d'eau à l'amont,

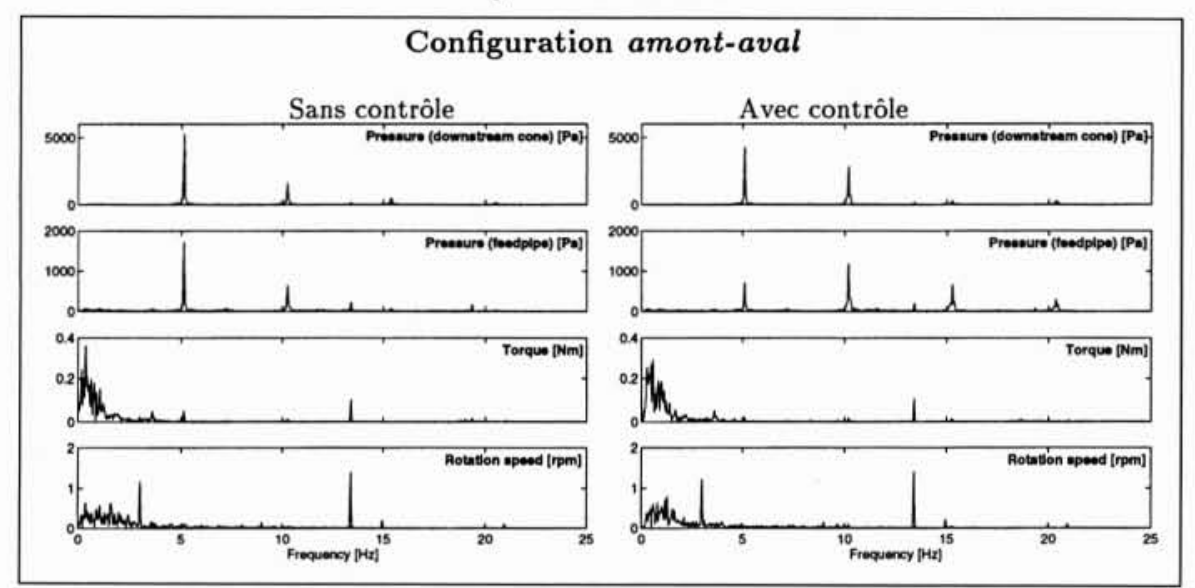

3. Trace fréquentielle pour la configuration prise d'eau à l'amont, injection à l'aval.

\section{IV — RESULTATS DES ESSAIS}

Pour chaque configuration, des mesures sont effectuées sans (s) ou avec (a) excitation externe contrôlée. L'efficacité est exprimée par le rapport (r) des amplitudes, à une fréquence donnée, de différentes grandeurs mesurées.

Remarquons que les résultats mentionnés ci-dessous prennent en considération l'ensemble des mesures effectuées et non seulement celles présentées dans les figures.

\subsection{Analyse des spectres}

Le tableau 2 donne, pour chacune des configurations testées, les amplitudes des fluctuations de pression à l'entrée de la machine à la fréquence de précession et ses deux premières harmoniques, ainsi que les amplitudes des fluctuations de vitesse et de couple à la fréquence de précession.

Dans le cas de la prise d'eau à l'amont, passant par la pompe et injection à l'amont (configuration amont-amont), le système de contrôle actif est efficace au niveau de la tuyauterie haute pression. On note typiquement des réductions d'un injection à l'amont.

facteur 2 à 5 des fluctuations de pression à la fréquence de précession. Par contre, il y a une nette augmentation des fluctuations de vitesse et de couple ainsi que de la première et/ou deuxième harmonique des fluctuations de pression. Toutefois, il est nécessaire de considérer non seulement les rapports $r=s / a$, mais également les amplitudes absolues respectives. On note des fluctuations de vitesses d'environ $2 \mathrm{tpm}$, soit $0,3 \%$ de la vitesse nominale, et de fluctuations de couple de $0,8 \mathrm{Nm}$, soit $0,1 \%$ du couple nominal. Bien que les fluctuations restent relativement petites en valeur absolue, elles impliquent des oscillations de la puissance générée par la turbine, ce qui n'est pas acceptable pour les exploitants. La puissance mécanique oscillatoire n'a pas été mesurée en tant que grandeur complexe.

Avec injection dans l'aspirateur et prise d'eau à l'amont, la pompe d'injection a été retirée du circuit d'excitation (configuration amont-aval) : la chute disponible au bornes de la turbine est utilisée pour mettre l'eau dans l'accumulateur sous pression. Pour cette configuration, les fluctuations de pression à l'entrée de la machine sont réduites d'un facteur 2 à 3 . Les fluctuations de couple et de vitesse sont peu affectées, mais dans le sens d'une réduction.

Avec prise d'eau à l'aval et injection dans l'aspirateur, en utilisant à nouveau la pompe d'injection (configuration avalaval), les résultats obtenus sont similaires aux précédents. Ce dernier cas est cependant intéressant, car dans le cas d'une turbine prototype, il est pratiquement toujours possible d'implanter une prise d'eau à l'aval tandis qu'un piquage au niveau de la tuyauterie haute pression risque d'être plus délicat à effectuer.

Tableau 2.- Analyse des spectres.

\begin{tabular}{|c|cc|c|c|c|c|}
\hline & & $\Delta p_{\text {amont }}^{\text {fond. }}$ & $\Delta p_{\text {amont }}^{1^{\circ} \text { harm. }}$ & $\Delta p_{\text {amont }}^{2^{\circ} \text { harm. }}$ & $\Delta T$ & $\Delta \omega$ \\
\hline Alim. amont & $\mathrm{s}$ & 1340 & 600 & 100 & 0.113 & 0.33 \\
(pompe) & $\mathrm{a}$ & 720 & 400 & 50 & 0.750 & 2.08 \\
Exc. amont & $\mathrm{r}$ & 1.86 & 1.50 & 2.00 & 0.15 & 0.16 \\
\hline Alim. amont & $\mathrm{s}$ & 1720 & 640 & 70 & 0.055 & 0.12 \\
(bypass) & $\mathrm{a}$ & 700 & 1180 & 660 & 0.025 & 0.06 \\
Exc. aval & $\mathrm{r}$ & 2.46 & 0.54 & 0.11 & 2.20 & 2.00 \\
\hline Alim. amont & $\mathrm{s}$ & 1670 & 650 & 70 & 0.802 & 0.07 \\
(bypass) & $\mathrm{a}$ & 640 & 2100 & 950 & 0.027 & 0.07 \\
Exc. aval & $\mathrm{r}$ & 2.61 & 0.31 & 0.07 & 1.91 & 1.00 \\
\hline
\end{tabular}

Unités : $\Delta \mathrm{p}=[\mathrm{Pa}], \Delta \mathrm{T}=[\mathrm{Nm}], \Delta \omega=[\mathrm{rpm}] ;$ Remarque : $\mathrm{s}=$ sans contrôle, $\mathrm{a}=$ avec contrôle, $\mathrm{r}=$ rapport $\mathrm{s} / \mathrm{a}$ 


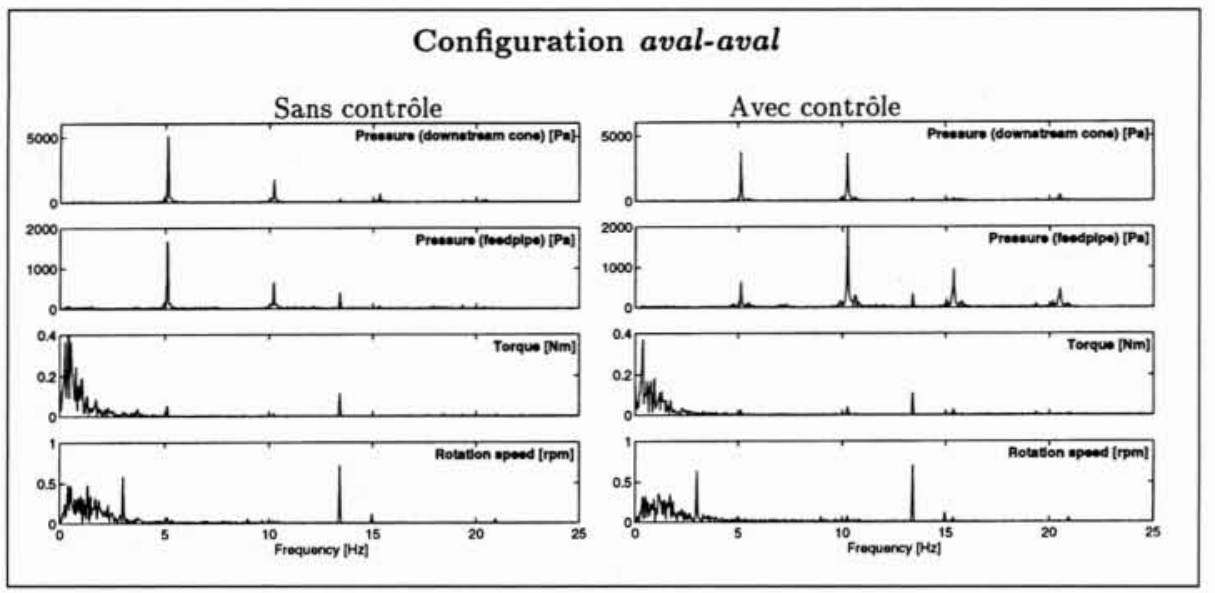

4. Trace fréquentielle pour la configuration prise d'eau à l'aval, injection à l'aval.

L'amplification de la première et/ou deuxième harmonique ne semble pas seulement être causée par l'excitatrice, mais est également liée aux conditions de fonctionnement de la turbine. Les résultats montrent également une bonne répétitivité pour un régime de fonctionnement donné.

\subsection{Bilan énergétique}

Le tableau 3 résume les puissances en jeu pour la turbine Francis et l'excitatrice.

Pour l'excitatrice, on distingue la puissance hydraulique consommée par la pompe en dérivation et le moteur de la vanne. Remarquons que pour la configuration amont-aval, la pompe n'est pas utilisée. Par contre, le débit injecté ne passe pas par la turbine et constitue donc une perte énergétique qui doit être incluse dans le bilan énergétique.

Globalement, le rapport de puissance $\mathrm{P}_{\text {excitatrice }} / \mathrm{P}$ turbine inférieur à $1 \%$. Plusieurs éléments dans la chaine de contrôle peuvent encore être optimisés, ce qui nous fait croire que ce rapport de puissance peut encore être amélioré. Vu que l'algorithme utilisé pour piloter l'excitatrice ne pouvait pas garantir une efficacité optimale durant toute la période de mesure, les performances restent relativement modestes.

Pour les configurations amont-aval et aval-aval, le rendement énergétique du système est moins bon que dans le cas amont-amont. Cela est principalement dû au fait que le débit injecté augmente considérablement (tableau 3). En effet, à cause de la présence d'un volume de vapeur dans l'aspirateur, un débit plus élevé est nécessaire pour obtenir une fluctuation de pression donnée.

\section{$\mathrm{V} \square$ CONCLUSION ET PERSPECTIVES}

Une étude expérimentale est réalisée à l'aide d'une excitatrice à vanne rotative. Le système est monté sur un modèle réduit de turbine Francis, fonctionnant à un régime de fluctuations hydrauliques établi. Cette expérience de faisabilité a été effectuée sur les plates-formes d'essai du laboratoire IMHEF-LMH. Elle a permis :

- de montrer l'efficacité d'un système de contrôle actif dans la réduction des fluctuations de pression dans le circuit,

— de vérifier le fonctionnement correct de l'actionneur,

- de faire un bilan énergétique,

- d'étudier l'influence de différentes positions de la prise d'eau et différentes positions d'insertion de l'actionneur dans la machine.

\section{Références}

[1] Grein, H. "Vibration phenomena in francis turbines : their causes and prevention". In Proc. of the 10th IAHR Symposium (Tokyo, September 1980), vol 1, pp. 527-539.

[2] Jacob, T., Blommaert, G., Prénat, J. E., Boyer, A. and Lauro, J.-F. "Essai d'amélioration de la stabilité de fonctionnement des turbines francis par le contrôle actif". In Proc. 7th Meeting of the AIRH Working Group on the Behaviour of Hydraulic Machinery under Steady osillator Conditions (Ljubljana, septembre 1995).

[3] Jacob, T. Evaluation sur modèle réduit et prédiction de la stabilité de fonctionnement des turbines francis, $\mathrm{PhD}$ thesis, Ecole Polytechnique Fédérale de Lausanne, 1993. Thèse EPFL n¹146.

Tableau 3. - Bilan énergétique.

\begin{tabular}{|c|c|c|c|c|c|}
\hline & Turbine & pompe (Turb.) & Vanne & $\mathrm{Q}_{\text {exci }} / \mathrm{Q}_{\text {turbine }}$ & $\mathrm{P}_{\text {excit }} / \mathrm{P}_{\text {turbine }}$ \\
\hline \multirow{3}{*}{$\begin{array}{l}\text { Alim. amont } \\
\text { (pompe) } \\
\text { Exc. amont }\end{array}$} & $\mathrm{E}=147$ & \multirow{3}{*}{$\begin{array}{l}E=164 \\
Q=0,0018 \\
P=0,30\end{array}$} & \multirow[b]{3}{*}{$\mathrm{P}=0,08$} & \multirow{3}{*}{$0,29 \%$} & \multirow{3}{*}{$0,41 \%$} \\
\hline & $Q=0,622$ & & & & \\
\hline & $\mathrm{P}=91,58$ & & & & \\
\hline \multirow{3}{*}{$\begin{array}{l}\text { Alim. amont } \\
\text { (bypass) } \\
\text { Exc. aval }\end{array}$} & $E=147$ & \multirow{3}{*}{$\begin{array}{l}E=147 \\
Q=0,0043 \\
P=0,63\end{array}$} & \multirow[b]{3}{*}{$P=0,29$} & \multirow{3}{*}{$0,68 \%$} & \multirow{3}{*}{$1,00 \%$} \\
\hline & $Q=0,623$ & & & & \\
\hline & $\mathrm{P}=91,59$ & & & & \\
\hline \multirow{3}{*}{$\begin{array}{l}\text { Alim. aval } \\
\text { (pompe) } \\
\text { Exc. aval }\end{array}$} & $E=147$ & \multirow{3}{*}{$\begin{array}{l}E=102 \\
Q=0,0052 \\
P=0,54\end{array}$} & \multirow[b]{3}{*}{$P=0,09$} & \multirow{3}{*}{$0,84 \%$} & \multirow{3}{*}{$0,59 \%$} \\
\hline & $Q=0,621$ & & & & \\
\hline & $\mathrm{P}=91,60$ & & & & \\
\hline
\end{tabular}

Unités : $\mathrm{E}=[\mathrm{J} / \mathrm{kg}], \mathrm{Q}=\left[\mathrm{m}^{3} / \mathrm{s}\right], \mathrm{P}=[\mathrm{kW}]$ 